\title{
Vector meson electromagnetic form factors
}

\author{
QCDSF Collaboration: M. Gürtler,, ${ }^{*}$ D. Brömmel, ${ }^{b}$ M. Göckeler, ${ }^{c}$ Ph. Hägler, ${ }^{a}$ \\ R. Horsley, ${ }^{d}$ Y. Nakamura, ${ }^{e}$ D. Pleiter, ${ }^{e}$ P.E.L. Rakow, ${ }^{f}$ A. Schäfer,${ }^{c}$ G. Schierholz, ${ }^{c, e}$ \\ H. Stüben ${ }^{g}$ and J.M. Zanotti ${ }^{d}$ \\ ${ }^{a}$ Institut für Theoretische Physik T39, Physik-Department der TU München, 85747 Garching, \\ Germany \\ ${ }^{b}$ School of Physics and Astronomy, University of Southampton, Southampton, SO17 1BJ, UK \\ ${ }^{c}$ Institut für Theoretische Physik, Universität Regensburg, 93040 Regensburg, Germany \\ ${ }^{d}$ School of Physics and Astronomy, University of Edinburgh, Edinburgh EH9 3JZ, UK \\ e John von Neumann-Institut für Computing NIC / DESY, 15738 Zeuthen, Germany \\ ${ }^{f}$ Theoretical Physics Division, Department of Mathematical Sciences, University of Liverpool, \\ Liverpool L69 3BX, UK \\ ${ }^{g}$ Konrad-Zuse-Zentrum für Informationstechnik Berlin, 14195 Berlin, Germany \\ Email: martin.guertler@ph.tum.de
}

\begin{abstract}
We present preliminary results for the electromagnetic form factors of vector mesons in lattice QCD. The analysis is based on two- and three-point functions, calculated with two-flavour clover-improved configurations for a lattice spacing of $a \sim 0.08 \mathrm{fm}$. The pions on these lattices are too heavy $\left(m_{\pi} \geq 400 \mathrm{MeV}\right)$ to allow for $\rho$ meson decay. We obtain a charge radius $\left\langle r^{2}\right\rangle \sim 0.49(5) \mathrm{fm}^{2}$, a $g$-factor $g_{\rho} \sim 1.6(1)$, and a small negative quadrupole moment $\mu_{Q}=-0.017(2) \mathrm{fm}^{2}$.
\end{abstract}

The XXVI International Symposium on Lattice Field Theory

July 14-19 2008

Williamsburg, Virginia, USA

\footnotetext{
*Speaker.
} 


\section{Introduction}

Most of our knowledge of the internal structure of hadrons is encoded in (generalised) form factors. They allow to study quantities like charge radii or multipole moments.

A number of lattice results is available for the nucleon, eg., [1] and the pion [2] electromagnetic form factors, and a first attempt in the quenched approximation to compute the form factors of vector mesons can be found in [3]. All these analyses are based on suitable hadron three-point functions. Another interesting ansatz based on density-density correlators can be found in [4]. A recent overview of the subject can be found in[5].

In this contribution, we present results for vector meson form factors in unquenched lattice QCD employing the standard three-point functions, obtained for non-perturbatively clover improved Wilson fermions and Wilson gauge action.

\section{Vector meson form factors}

We consider the interaction of a vector meson with an electromagnetic current $J^{\alpha}$. In this section, all quantities are expressed in Minkowski space, $g_{\mu \nu}=\operatorname{diag}(-1,1,1,1)$. The relevant matrix element $\left\langle p^{\prime}, s^{\prime}\left|J^{\alpha}\right| p, s\right\rangle$ can be parametrised

$$
\begin{aligned}
N\left\langle p^{\prime}, s^{\prime}\left|J^{\alpha}\right| p, s\right\rangle= & -G_{1}\left(Q^{2}\right)\left(\varepsilon^{* *} \cdot \varepsilon\right) P^{\alpha} \\
& -G_{2}\left(Q^{2}\right)\left[\left(\varepsilon^{\prime *} \cdot q\right) \varepsilon^{\alpha}-(\varepsilon \cdot q) \varepsilon^{* \alpha}\right] \\
& +G_{3}\left(Q^{2}\right)(\varepsilon \cdot q)\left(\varepsilon^{\prime *} \cdot q\right) P^{\alpha} /\left(2 m_{\rho}\right)^{2} .
\end{aligned}
$$

Here $s\left(s^{\prime}\right)$ is the spin and $p\left(p^{\prime}\right)$ the momentum of the incoming (outgoing) vector meson, $P=p+p^{\prime}$, and $N=2 \sqrt{E_{\rho}\left(\vec{p}^{\prime}\right) E_{\rho}(\vec{p})}$ is a normalisation factor. The meson's polarisation $\lambda \in\{0,+,-\}$ is encoded in the polarisation vectors $\varepsilon(\lambda, \vec{p})$, which obey $p^{\mu} \varepsilon_{\mu}(\vec{p}, \lambda)=0$ and the completeness relation

$$
\sum_{\lambda=0,+,-} \varepsilon_{i}(\vec{p}, \lambda)^{*} \varepsilon_{j}(\vec{p}, \lambda)=-g_{i j}+p_{i} p_{j} / m^{2} .
$$

The form factors $G_{i}$ depend on the momentum transfer $Q^{2}=-q^{2}=-\left(p^{\prime}-p\right)^{2}$. They can be rewritten in terms of the Sachs charge, magnetic and quadrupole form factors:

$$
\begin{aligned}
G_{C}\left(Q^{2}\right) & =G_{1}\left(Q^{2}\right)+2 / 3 \eta G_{Q}\left(Q^{2}\right) \\
G_{M}\left(Q^{2}\right) & =G_{2}\left(Q^{2}\right) \\
G_{Q}\left(Q^{2}\right) & =G_{1}\left(Q^{2}\right)-G_{2}\left(Q^{2}\right)+(1+\eta) G_{3}\left(Q^{2}\right),
\end{aligned}
$$

where $\eta=Q^{2} /\left(2 m_{\rho}\right)^{2}$. The momentum transfer dependence of the electric form factor $G_{C}$ contains information on the charge radius

$$
\left\langle r^{2}\right\rangle=-\left.6 \frac{\partial G_{C}}{\partial\left(Q^{2}\right)}\right|_{Q^{2}=0},
$$

while $G_{M}$ gives the magnetic moment of the $\rho$ meson

$$
\mu_{\rho}=\frac{e}{2 m_{\rho}} G_{M}\left(Q^{2}=0\right) .
$$


The forward limit of $G_{M} \equiv G_{2}$ itself is also called the $\rho$ meson's $g$-factor $g_{\rho}$. In the simulation, it can be accessed only from an extrapolation in $q^{2} \rightarrow 0$ because the $G_{2}$ contribution in Eq. (2.1) vanishes in the forward case. Information about the shape of the vector meson is encoded in the quadrupole moment $\mu_{Q}=e / m_{\rho}^{2} G_{Q}(0)$; for $\mu_{Q}<0$ the meson is oblate, and for $\mu_{Q}>0$ its shape is prolate. Also $\mu_{Q}$ can only be determined from a $q^{2} \rightarrow 0$ extrapolation.

\section{Lattice calculation}

On the lattice, the calculation of form factors requires an analysis of suitable three-point functions. A meson with momentum $\vec{p}$ is created at time $t=0$, interacts with the electromagnetic current at intermediate time $\tau$, and gets annihilated at time $t=t_{\text {sink }}$. Its creation is described by an interpolating operator $\chi_{\mu}^{\dagger}=\bar{d} \gamma_{\mu} u$, which has non-vanishing overlap with a charged $\rho$ meson:

$$
\left\langle\Omega\left|\chi_{\mu}(0)\right| \rho(\vec{p}, s)\right\rangle={\sqrt{2 E_{\rho}(\vec{p})}}^{-1} \lambda_{\rho}(\vec{p}) \varepsilon_{\mu}(p, s) .
$$

Here, $\lambda_{\rho}$ describes the overlap of $\chi_{\mu}$ with the $\rho$ meson, and $\varepsilon_{\mu}$ is the polarisation vector. The corresponding three-point function is given by

$$
G_{\mu \nu}^{\alpha}\left(t, \tau, \vec{p}^{\prime}, \vec{p}\right)=\sum_{\vec{x}, \vec{\xi}} e^{-i \vec{p}^{\prime}(\vec{x}-\vec{\xi})} e^{-i \vec{p} \vec{\xi}}\left\langle\Omega\left|\chi_{\mu}(x) J^{\alpha}(\xi) \chi_{v}^{\dagger}(0)\right| \Omega\right\rangle
$$

which can be written for large time separations as

$$
\begin{aligned}
\lim _{\substack{\tau \rightarrow \infty \\
t-\tau \rightarrow \infty}} G_{\mu \nu}^{\alpha}\left(t, \tau, \vec{p}^{\prime}, \vec{p}\right)= & \frac{e^{-E_{\rho}\left(\vec{p}^{\prime}\right)(t-\tau)} e^{-E_{\rho}(\vec{p}) \tau}}{4 E_{\rho}\left(\vec{p}^{\prime}\right) E_{\rho}(\vec{p})} \lambda_{\rho}\left(\vec{p}^{\prime}\right) \bar{\lambda}_{\rho}(\vec{p}) \\
& \times\left(g_{\mu \tau}-\frac{p_{\mu}^{\prime} p_{\tau}^{\prime}}{m_{\rho}^{2}}\right) J^{\tau \alpha \sigma}\left(g_{\sigma v}-\frac{p_{\sigma} p_{v}}{m_{\rho}^{2}}\right),
\end{aligned}
$$

where we have neglected the exponentially suppressed contributions from excited states, and where $J^{\tau \alpha \sigma}$ is defined via $J^{\alpha}=\varepsilon_{\tau}^{* *} J^{\tau \alpha \sigma} \varepsilon_{\sigma}$. Two-point functions are defined by

$$
G_{\mu v}(t, \vec{p})=\sum_{\vec{x}} e^{-i \vec{p} \vec{x}}\left\langle\Omega\left|\chi_{\mu}(x) \chi_{v}^{\dagger}(0)\right| \Omega\right\rangle .
$$

For a sufficiently large separation of source and sink they read

$$
\lim _{t \rightarrow \infty} G_{\mu v}(t, \vec{p})=-\frac{e^{-E_{\rho}(\vec{p}) t}}{2 E_{\rho}(\vec{p})} \lambda_{\rho}(\vec{p}) \bar{\lambda}_{\rho}(\vec{p})\left(g_{\mu v}-\frac{p_{\mu} p_{v}}{m_{\rho}^{2}}\right) .
$$

Consequently, the overlap factors $\lambda_{\rho}$ and the exponentials from Eqs. (3.1) and (3.2) cancel in the ratio

$$
R_{\mu \nu}^{\alpha}\left(\tau, \vec{p}^{\prime}, \vec{p}\right)=\frac{G_{\mu \nu}^{\alpha}\left(t, \tau, \vec{p}^{\prime}, \vec{p}\right)}{G_{\mu \mu}\left(t, \vec{p}^{\prime}\right)} \sqrt{\frac{G_{v v}(t-\tau, \vec{p}) G_{\mu \mu}\left(\tau, \vec{p}^{\prime}\right) G_{\mu \mu}\left(t, \vec{p}^{\prime}\right)}{G_{v v}(\tau, \vec{p}) G_{\mu \mu}\left(t-\tau, \vec{p}^{\prime}\right) G_{\nu v}(t, \vec{p})}}
$$

which is independent of $\tau$ for large time separations. Thus, we obtain a system of linear equations

$$
R_{\mu v}^{\alpha}\left(\vec{p}, \vec{p}^{\prime}\right)=\sum_{i} c_{i} G_{i}
$$




\begin{tabular}{|c|c|c|c|c|c|c|}
\hline Volume & $\beta$ & $\kappa$ & $m_{\pi}[\mathrm{MeV}]$ & $m_{\rho}[\mathrm{MeV}]$ & $a[\mathrm{fm}]$ & $t_{\text {sink }}$ \\
\hline $16^{3} 32$ & 5.29 & 0.13500 & $929(2)$ & $1228(4)$ & 0.089 & 14 \\
$16^{3} 32$ & 5.29 & 0.13550 & $784(3)$ & $1090(8)$ & 0.084 & 14 \\
$24^{3} 48$ & 5.29 & 0.13590 & $591(2)$ & $965(6)$ & 0.080 & 18 \\
$24^{3} 48$ & 5.29 & 0.13620 & $406(6)$ & $898(10)$ & 0.077 & 18 \\
\hline
\end{tabular}

Table 1: Compilation of parameters.

with easily computable coefficients $c_{i}$ of the $G_{i}$ for each value of $Q^{2}$. This overdetermined system is solved by $\chi^{2}$ minimisation to finally obtain the form factors $G_{i}$.

For our computation, we use QCDSF configurations, generated with two flavours of clover improved Wilson fermions and the Wilson gauge action. The improvement coefficient $c_{S W}$ was determined non-perturbatively[6], giving $c_{S W}=1.9192$ at $\beta=5.29$. Parameters, lattice constants etc. are summarised in Tab. 1. The scale was set using the nucleon mass. Jacobi smearing is applied ( $\kappa=0.21, N=60)$ in order to enhance the ground state overlap of our source and sink operators.

We use the sequential source technique for the calculation of the three-point functions. The sink time $t_{\text {sink }}$ is fixed to the values in Tab. 1 , where the source for the secondary inversion is constructed from the quark propagator. Without further inversions, three point functions with insertions of arbitrary operators on all time slices can be computed.

Because each value of the sink momentum $\vec{p}^{\prime}=2 \pi / L_{s} \vec{n}^{\prime}$ ( $L_{s}$ being the spatial lattice extent) requires separate secondary inversions and is thus expensive, we use only three values for $\vec{n}^{\prime}$, namely $(0,0,0),(-1,0,0)$, and $(0,-1,0)$. For the source momentum, we take $2 \pi / L_{s} \vec{n}$, with $\vec{n}$ assuming $(0,0,0),(1,0,0),(1,1,0),(1,1,1),(2,0,0),(2,1,1),(2,2,1)$ and all possible permutations of the vector components.

There is no need to consider the disconnected part of the three-point functions, because their contributions from a gauge configuration $U$ and its complex conjugate $U^{*}$ (which have the same weight) cancel in the ensemble average.

The physical $\rho$ meson is a resonance and decays predominantly into two pions. In contrast, the vector mesons on the lattices we consider here are stable, because their mass is too small to create two pions in a relative $P$ wave. This means that the quantities we compute here are not directly accessible to any experiment, and should rather be considered as being exemplary for a generic spin-1 particle.

\section{Results}

Fig. 1 shows the Sachs form factors (2.2) for the smallest pion mass we have analysed so far. On this lattice, the smallest momentum transfer we have is $Q_{\min }^{2} \sim 0.44 \mathrm{GeV}^{2}$. This is relatively large, and will be improved in the future by the use of partially twisted boundary conditions [7].

The electric form factor is fitted with the phenomenologically motivated monopole ansatz

$$
G_{C}\left(Q^{2}\right)=\frac{G_{C}(0)}{1+Q^{2} / m_{C}^{2}} .
$$

In the forward case, $Q^{2}=0$, this is equal to the electric charge. Because we use the non-conserved local vector current $\gamma^{\mu}$, then measured electric form factor in the forward case $G_{C}^{\text {unren }}(0)$ gives us 


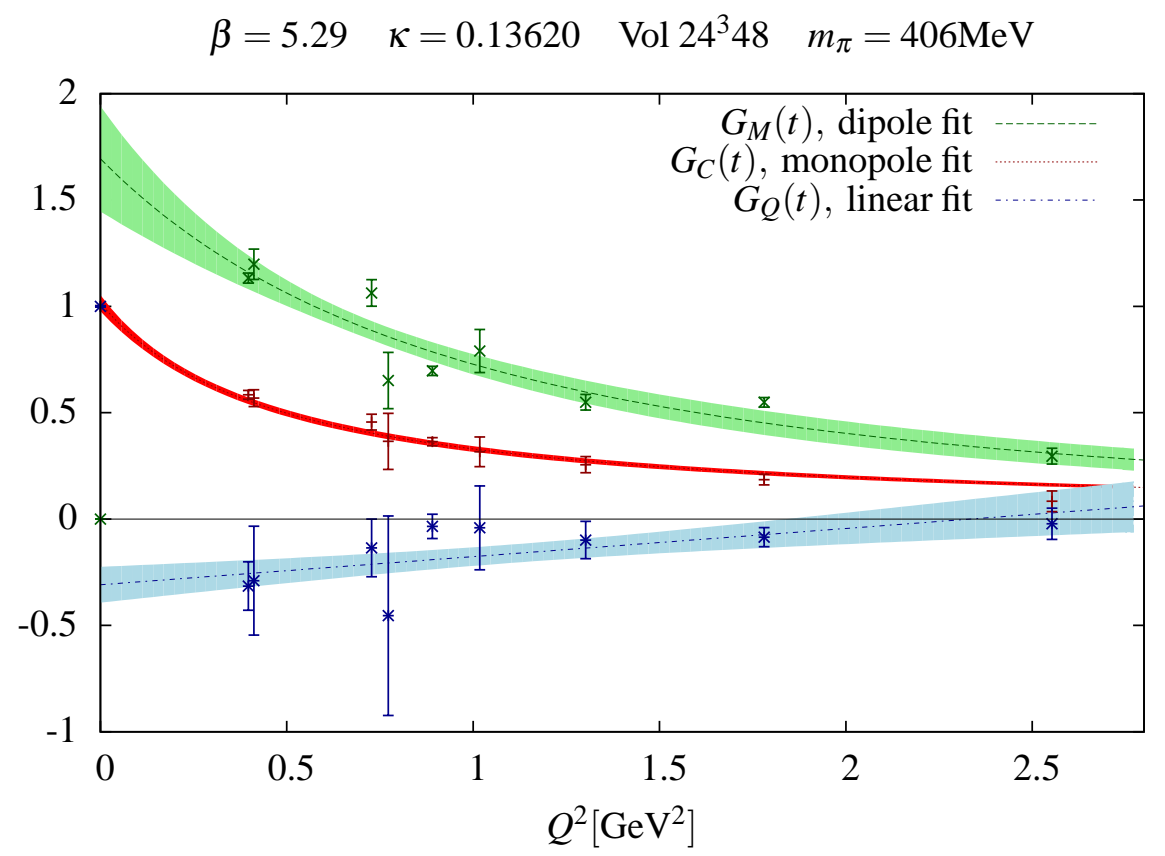

Figure 1: Example plot of form factors, together with fits described in Sec. 4.

the renormalisation factor $Z_{V}=1 / G_{C}^{\text {unren }}(0)$, that has to be applied to all three-point functions. However, we do not fix $G_{C}(0)$ in the monopole fit. The charge radius is computed according to Eq. (2.3).

For the magnetic form factor, a monopole ansatz would be inadequate, because this would lead to divergencies in the magnetisation density one obtains after Fourier transforming to coordinate space. Thus, we use a dipole ansatz

$$
G_{M}\left(Q^{2}\right)=\frac{G_{M}(0)}{\left(1+Q^{2} / m_{M}^{2}\right)^{2}} .
$$

The quadrupole form factor shows relatively large error bars, and is for all but the smallest non-vanishing momentum transfer compatible with zero. To get a first idea about the quadrupole moment, we use an ansatz linear in $Q^{2}$ to extrapolate the lattice data to $Q^{2}=0$. An analysis with partially twisted boundary conditions will be helpful here, too, in order to decide about the magnitude of the quadrupole moment.

The results for charge radii, magnetic and quadrupole moment for all analysed lattices are summarised in table 2. In Fig. 2 we plot these results as functions of $m_{\pi}^{2}$ and attempt a linear extrapolation to the chiral limit. The (squared) charge radius $\left\langle r^{2}\right\rangle$ behaves as one would expect from the pion cloud picture, showing the growth of the pion cloud with decreasing quark mass. The value of the charge radius is similar to the value known from the nucleon, and larger than the pion charge radius. This would also be the expectation from quark models, that attribute the larger size of a vector meson to the repulsive hyperfine interaction of the aligned spins, whereas the anti-aligned spins in the pion experience hyperfine attraction. Our linear extrapolation gives a charge radius of $\left\langle r^{2}\right\rangle=0.49(5) \mathrm{fm}^{2}$. 


\begin{tabular}{|cc|c|c|c|}
\hline$\kappa$ & volume & $\left\langle r^{2}\right\rangle\left[\mathrm{fm}^{2}\right]$ & $\mu_{M}$ & $\mu_{Q}\left[\mathrm{fm}^{2}\right]$ \\
\hline 0.13500 & $16^{3} 32$ & $0.33(5)$ & $2.1(3)$ & $0.002(5)$ \\
0.13550 & $16^{3} 32$ & $0.42(8)$ & $1.7(3)$ & $-0.004(2)$ \\
0.13590 & $24^{3} 48$ & $0.38(3)$ & $1.8(2)$ & $-0.007(3)$ \\
0.13620 & $24^{3} 48$ & $0.49(4)$ & $1.7(3)$ & $-0.015(4)$ \\
\hline
\end{tabular}

Table 2: Charge radii, magnetic and quadrupole moments on the analysed lattices.

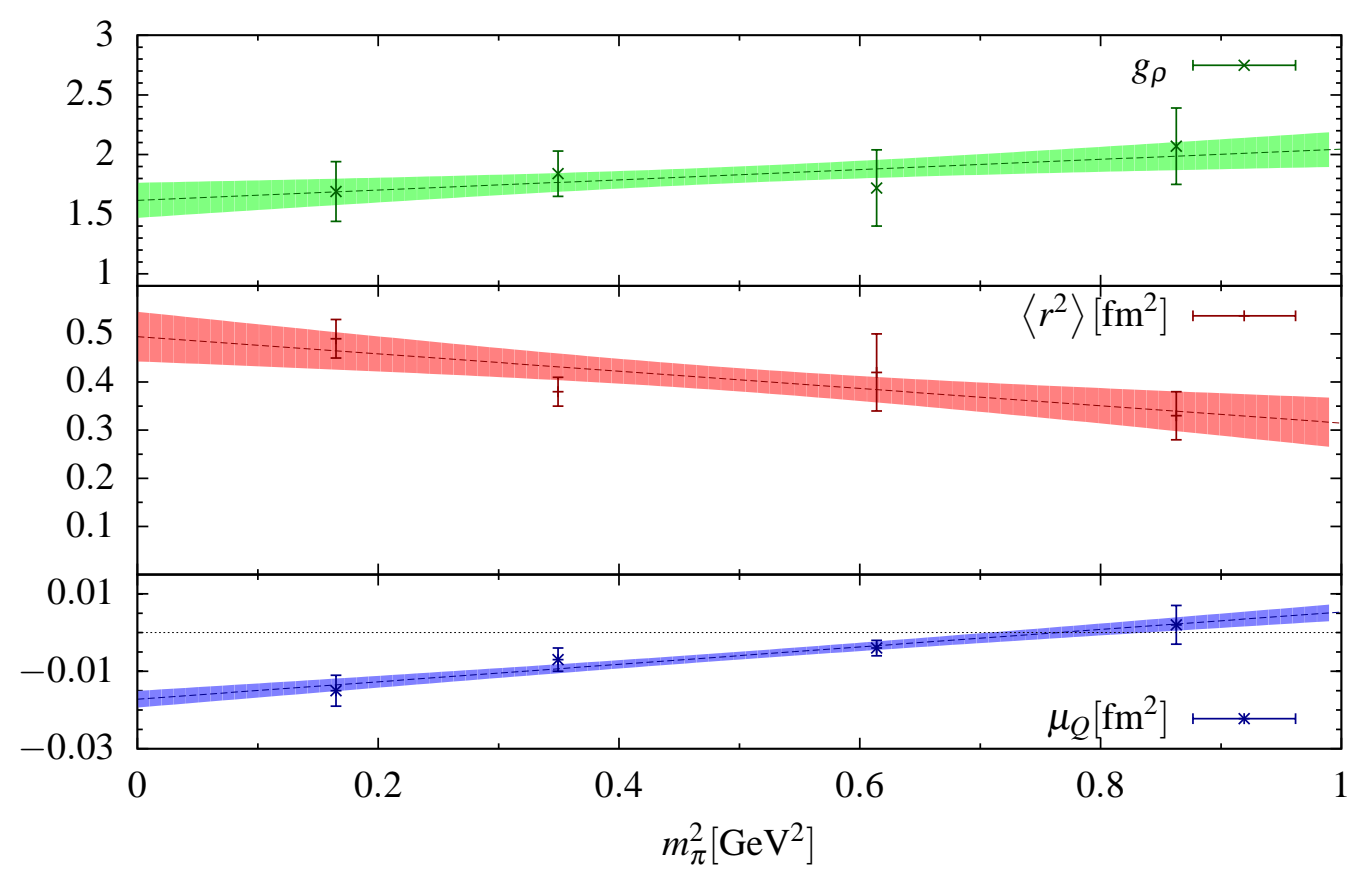

Figure 2: Chiral extrapolation for charge radius, $g$-factor and quadrupole moment.

For the $g$-factor we obtain $g_{\rho}=1.6(1)$. This is compatible with expectations based on sum rule calculations, $g_{\rho}=2.0(3)$ [8]. The quark model calculation in [9] gives $g_{\rho}=1.92$, and [10] obtain $g_{\rho}=2.01$, based on Dyson-Schwinger equations. It is considerably smaller than the quenched lattice result $g_{\rho} \sim 2.3$ of [3]. Their result, however, was not based on an extrapolation in $Q^{2}$, but on an estimate assuming $G_{C} \propto G_{M}$, using only one non-zero value of the momentum transfer.

A phenomenologically interesting quantity is the quadrupole moment. For large quark masses, and for large momentum transfers, the quadrupole form factor is hardly distinguishable from zero. For small quark masses, however, we see a trend of $G_{Q}$ becoming increasingly negative, being clearly different from zero for our lightest pion and small values of the momentum transfer. The linear extrapolation gives a value of $\mu_{Q}=-0.017(2) \mathrm{fm}^{2}$. The negative sign is interpreted as the $\rho$ meson having oblate shape, an observation also found in [3], and [4]. The statistical uncertainty, and the systematic uncertainties from the extrapolations to the forward limit are still rather large. Our results should therefore be considered as preliminary. Calculations at smaller quark masses and smaller values of the momentum transfer using, e.g., partially twisted boundary conditions, are 
clearly desirable to reach firm conclusions.

\section{Summary}

We have computed the electromagnetic vector meson form factors in a dynamical two-flavour lattice simulation. We determined the charge radius of the $\rho$ meson from a monopole fit of the $q^{2}$ dependence of the electric form factor, obtaining $\left\langle r^{2}\right\rangle=0.49(5) \mathrm{fm}^{2}$ from a linear extrapolation to vanishing quark mass. From a dipole extrapolation of the magnetic form factor, we obtained a $g$-factor of $g_{\rho}=1.6(1)$ as forward limit of the magnetic form factor. The result for the quadrupole form factor implies an oblate shape of the $\rho$ meson, manifesting itself in a quadrupole moment of $\mu_{Q}=-0.017(2) \mathrm{fm}^{2}$. We are currently improving our study by repeating the analysis for more ensembles and by employing partially twisted boundary conditions.

\section{Acknowledgement}

The numerical calculations have been performed on the Hitachi SR8000 at LRZ (Munich), the APE1000 and apeNEXT at NIC/DESY (Zeuthen) and the BlueGeneL's at NIC/Jülich and EPCC. This work was supported by the DFG (Forschergruppe Gitter-Hadronen- Phänomenologie, Transregional Collaborative Research Centre SFB/TRR 55 and Emmy-Noether program) and by the EU I3HP under contract number RII3-CT-2004-506078. P. H. thanks the Excellence Cluster Universe at Technische Universität München for support.

\section{References}

[1] M. Göckeler et al. [QCDSF/UKQCD Collaboration], PoS LAT2007 (2007) 161 [arXiv:0710.2159 [hep-lat]].

[2] D. Brömmel et al. [QCDSF/UKQCD Collaboration], Eur. Phys. J. C 51 (2007) 335 [arXiv:hep-lat/0608021].

[3] J. N. Hedditch, W. Kamleh, B. G. Lasscock, D. B. Leinweber, A. G. Williams and J. M. Zanotti, Phys. Rev. D 75 (2007) 094504 [arXiv:hep-lat/0703014].

[4] C. Alexandrou, P. de Forcrand and A. Tsapalis, Phys. Rev. D 66 (2002) 094503 [arXiv:hep-lat/0206026], C. Alexandrou and G. Koutsou, arXiv:0809.2056 [hep-lat].

[5] J. Zanotti, PoS LATTICE 2008 (2008) 007

[6] K. Jansen and R. Sommer [ALPHA collaboration], Nucl. Phys. B 530 (1998) 185 [Erratum-ibid. B 643 (2002) 517] [arXiv:hep-lat/9803017].

[7] C. T. Sachrajda and G. Villadoro, Phys. Lett. B 609 (2005) 73 [arXiv:hep-lat/0411033].

[8] A. Samsonov, JHEP 0312 (2003) 061 [arXiv:hep-ph/0308065].

[9] H. M. Choi and C. R. Ji, Phys. Rev. D 70 (2004) 053015 [arXiv:hep-ph/0402114].

[10] M. S. Bhagwat and P. Maris, Phys. Rev. C 77 (2008) 025203 [arXiv:nucl-th/0612069]. 